\title{
An inductive construction for Hamilton cycles in Kneser graphs
}

\author{
J. Robert Johnson \\ School of Mathematical Sciences \\ Queen Mary University of London, E1 4NS, UK \\ r.johnson@qmul.ac.uk. \\ Submitted: June 22, 2009; Accepted: Sep 9, 2011; Published: Sep 20, 2011 \\ Mathematics Subject Classification: 05C38, 05C45
}

\begin{abstract}
The Kneser graph $K(n, r)$ has as vertices all $r$-subsets of an $n$-set with two vertices adjacent if the corresponding subsets are disjoint. It is conjectured that, except for $K(5,2)$, these graphs are Hamiltonian for all $n \geq 2 r+1$. In this note we describe an inductive construction which relates Hamiltonicity of $K(2 r+2 s, r)$ to Hamiltonicity of $K\left(2 r^{\prime}+s, r^{\prime}\right)$. This shows (among other things) that Hamiltonicity of $K(2 r+1, r)$ for all $3 \leq r \leq k$ implies Hamiltonicity of $K(2 r+2, r)$ for all $r \leq 2 k+1$. Applying this result extends the range of values for which Hamiltonicity of $K(n, r)$ is known. Another consequence is that certain families of Kneser graphs $\left(K\left(\frac{27}{13} r, r\right)\right.$ for instance) contain infinitely many Hamiltonian graphs.
\end{abstract}

\section{Introduction}

Let $S$ be a finite set. We write $K(S, r)$ for the graph with vertex set $S^{(r)}=\{X \subseteq$ $S:|X|=r\}$, and two vertices adjacent if the corresponding sets are disjoint. Up to isomorphism $K(S, r)$ depends only on the cardinality of $S$ and so for $n \in \mathbb{N}$ we write $K(n, r)$ for $K(\{1,2, \ldots, n\}, r)$.

The graphs $K(n, r)$ are known as Kneser graphs. It is conjectured that $K(n, r)$ is Hamiltonian for all pairs $(n, r)$ with $n \geq 2 r+1$ except for $(5,2)$ (this is Petersen' $\mathrm{s}$ graph). However, this is only known in general when $n$ is much larger than $2 r+1$. Specifically, Chen [2] showed that $K(n, r)$ is Hamiltonian whenever

$$
n \geq \frac{3 r+1+\sqrt{5 r^{2}-2 r+1}}{2}=(2.62 \cdots+o(1)) r .
$$

Hamiltonicity is also known for several small values (see [3] and references therein). The strongest results in this direction being that $K(n, r)$ is Hamiltonian for all $n \leq 27$, proved by Savage and Shields [3] using a computer search. 
Intuitively the graph $K(2 r+s, r)$ should be more likely to be Hamiltonian when $s$ is large since it has higher degree and higher edge density then. However, it is not obvious that this is true. For instance, does the Hamiltonicity of the family of graphs $K(2 r+2, r)$ follow from the Hamiltonicity of the family of graphs $K(2 r+1, r)$ (this intriguing family of Kneser graphs is known as the odd graphs [1])?

We describe a construction which proves this and more:

Theorem 1. Let $r \geq 3$ and $n=2 r+s$ be even. If $K\left(2 a+\frac{s}{2}, a\right)$ is either Hamiltonian or $K(5,2)$ for all $1 \leq a \leq\left\lfloor\frac{r}{2}\right\rfloor$ then $K(n, r)$ is Hamiltonian.

The exception made for $K(5,2)$ is clearly needed since Petersen's graph is notoriously non-Hamiltonian. In our constructions we get round this by using a Hamilton path in $K(5,2)$ and modifying our arguments slightly.

We give two appealing corollaries of our main theorem.

Corollary 2. If $K(2 r+1, r)$ is Hamiltonian for all $3 \leq r \leq k$ then $K(2 r+2, r)$ is Hamiltonian for all $3 \leq r \leq 2 k+1$. In particular $K(2 r+2, r)$ is Hamiltonian for all $r \leq 27$.

It appears that the best previously known result is that $K(2 r+2, r)$ is Hamiltonian when $r \leq 13$ [3].

Proof. This follows immediately from Theorem 1 with $s=2$ and the fact that $K(2 r+1, r)$ is Hamiltonian for all $3 \leq r \leq 13$ [3].

Corollary 3. If $K(2 r+1, r)$ is Hamiltonian for all $3 \leq r \leq k$ then $K\left(\frac{2 k+1}{k} r, r\right)$ is Hamiltonian for infinitely many values of $r$.

A particular consequence of this and the fact that $K(2 r+1, r)$ is Hamiltonian for all $3 \leq r \leq 13[3]$ is that $K\left(\frac{27}{13} r, r\right)$ is Hamiltonian for infinitely many $r$.

Proof. We claim that under the assumptions given the graph $K\left(2 r+2^{l}, r\right)$ is Hamiltonian for all $l \geq 0,3 \leq r \leq k 2^{l}$. Given this claim, setting $r=k 2^{l}$ provides infinitely many Hamiltonian graphs in $K\left(\frac{2 k+1}{k} r, r\right)$.

We will prove the claim by induction on $l$. The base case $l=0$ is the statement that $K(2 r+1, r)$ for all $3 \leq r \leq k$. For the induction step we may assume that $K\left(2 r+2^{l-1}, r\right)$ is Hamiltonian for all $3 \leq r \leq k 2^{l-1}$ which, by Theorem 1, implies that $K\left(2 r+2^{l}, r\right)$ is Hamiltonian for all $3 \leq r \leq k 2^{l}$.

\section{Notation}

For $a, b \in \mathbb{N}$ we write $[a, b]$ for $\{a, a+1, \ldots, b\}$ and $[a]$ for $[1, a]$.

The key idea is to relate the graphs $K(n, r)$ and $K\left(\frac{n}{2}, a\right)$ by splitting the ground set $[n]$ into $\frac{n}{2}$ pairs, and considering how an $r$-subset of $[n]$ intersects these. To this end we need 
some notation. Let $n=2 m$ be even. Let $p_{i}=\{2 i-1,2 i\}$ so that the sets $p_{1}, p_{2}, \ldots, p_{m}$ partition $[n]$. If $X \subseteq[n]$ we define:

$$
X(i)=\left\{\begin{aligned}
0 & \text { if } X \cap p_{i}=\emptyset \\
-1 & \text { if } X \cap p_{i}=\{2 i-1\} \\
1 & \text { if } X \cap p_{i}=\{2 i\} \\
2 & \text { if } X \cap p_{i}=p_{i}
\end{aligned}\right.
$$

and identify $X$ with the $m$-tuple $(X(1), X(2), \ldots, X(m)) \in\{-1,0,1,2\}^{m}$.

We also define

$$
\begin{aligned}
& A(X)=\{i \in[m]: X(i)=2\} \\
& B(X)=\{i \in[m]: X(i)= \pm 1\}
\end{aligned}
$$

and

$$
f_{X}: B(X) \rightarrow\{ \pm 1\}
$$

to be the function with $f_{X}(i)=X(i)$.

It is clear that $A(X) \cap B(X)=\emptyset$ and $|X|=2|A(X)|+|B(X)|$. Moreover, the triple $\left(A(X), B(X), f_{X}\right)$ determines the set $X$. In what follows it will be convenient to swap between the representation of a set $X$ by the $X(i)$ and by $\left(A(X), B(X), f_{X}\right)$.

Let $S_{a, b}=\{X \subseteq[n]:|A(X)|=a,|B(X)|=b\}$. It is clear that

$$
[n]^{(r)}=\bigcup_{a=0}^{\left\lfloor\frac{r}{2}\right\rfloor} S_{a, r-2 a},
$$

and that this is a disjoint union. When $a$ is an integer we denote by $K_{a}(n, r)$ the subgraph of $K(n, r)$ induced by $S_{a, r-2 a}$. When $A$ is a set of integers we denote by $K_{A}(n, r)$ the subgraph of $K(n, r)$ induced by $\bigcup_{a \in A} S_{a, r-2 a}$.

A permutation of $[n]$ induces an automorphism of the graph $K(n, r)$. If the permutation preserves the partition of $[n]$ into the pairs $p_{1}, \ldots, p_{m}$ then the automorphism induced preserves the sets $S_{a, r-2 a}$. In what follows when we refer to permuting or reordering the pairs $p_{1}, \ldots, p_{m}$ we mean applying such an automorphism.

\section{Construction}

Throughout $n=2 m$ will be even. Our strategy is to find cycles in each of $K_{a}(n, r)$ and then to join them up. Specifically, we first construct a Hamilton cycle $\mathcal{H}_{0}$ in $K_{0}(n, r)$. Next we partition each of $K_{a}(n, r) a \geq 1$ into a small number of cycles. Finally, we join the cycles which partition $K_{1}(n, r)$ to $\mathcal{H}_{0}$, join the cycles which partition $K_{2}(n, r)$ to this new cycle, and so on until we have a Hamilton cycle in $K_{\left\{0,1, \ldots,\left\lfloor\frac{r}{2}\right\rfloor\right\}}(n, r)=K(n, r)$.

The existence of $\mathcal{H}_{0}$ is the content of Lemma 1 . The partition of $K_{a}(n, r)$ into cycles is the content of Lemma 2. In each case we need some extra properties to carry out the joining process.

If $c \in\{ \pm 1\}^{k}$ we denote the $k$-tuple $-c$ by $\bar{c}$. 
Lemma 1. For all $r \geq 3$ and even $n \geq 2 r+2$ there is a Hamilton cycle $\mathcal{H}$ in $K_{0}(n, r)$ with the property that for every $c \in\{ \pm 1\}^{r-2}$ there is an edge $X Y$ of $\mathcal{H}$ with

$$
\begin{aligned}
& X=(c, 0, \pm 1, \pm 1, \underbrace{0, \ldots}_{\underbrace{0, \ldots, 0}_{m-r-1}}) \\
& Y=(\bar{c}, \pm 1,0, \pm 1, \underbrace{0, \ldots, 0}_{m-r-1}) .
\end{aligned}
$$

Lemma 2. Let $n=2 m$ and $1 \leq a \leq\left\lfloor\frac{r}{2}\right\rfloor$ with $(n, r, a) \neq(10,4,2)$. If the graph $K(2 a+$ $m-r, a)$ is Hamiltonian or is $K(5,2)$ then the graph $K_{a}(n, r)$ can be partitioned into cycles $C_{1}, C_{2}, \ldots, C_{k}$ where $k \leq 2^{r-2 a}$. Moreover, this can be done in such a way that:

1. for $1 \leq i \leq k$, each of the cycles $C_{i}$ contains an edge $X Y$ with

$$
\begin{aligned}
& X=(c(i), \underbrace{2, \ldots, 2}_{a}, \underbrace{0, \ldots, 0}_{a}, \underbrace{0, \ldots, 0}_{m-r}) \\
& Y=(\overline{c(i)}, \underbrace{0, \ldots, 0}_{a}, \underbrace{2, \ldots, 2}_{a}, \underbrace{0, \ldots, 0}_{m-r})
\end{aligned}
$$

where $c(1), c(2), \ldots, c(k)$ are distinct elements of $\{ \pm 1\}^{r-2 a}$, and

2. there is some reordering of the pairs $p_{1}, \ldots, p_{m}$ with respect to which for every $d \in$ $\{ \pm 1\}^{r-2 a-2}$ there is an edge $X Y$ in one of the $C_{i}$ with

$$
\begin{aligned}
& X=(d, 0, \underbrace{0, \ldots, 0}_{a}, \pm 1, \underbrace{2, \ldots, 2}_{a}, \pm 1, \underbrace{0, \ldots, 0}_{m-r-1}) \\
& Y=(\bar{d}, \pm 1, \underbrace{2, \ldots, 2}_{a}, 0, \underbrace{0, \ldots, 0}_{a}, \pm 1, \underbrace{0, \ldots, 0}_{m-r-1}) .
\end{aligned}
$$

We postpone the proofs of these until the next section and move on to using them to prove Theorem 1.

We repeatedly use the concept of inserting one cycle in another. Suppose that we have two vertex disjoint cycles

$$
\begin{aligned}
& X=x_{1}, x_{2}, \ldots, x_{k} \\
& Y=y_{1}, y_{2}, \ldots, y_{l}
\end{aligned}
$$

in a graph $G$. If, for some $i, j$, we have $x_{i} y_{j}, x_{i+1} y_{j+1} \in E(G)$ then we can form a new cycle by adding these edges and removing the edges $x_{i} x_{i+1}$ and $y_{j} y_{j+1}$. Specifically, this new cycle is

$$
x_{1}, x_{2}, \ldots, x_{i-1}, x_{i}, y_{j}, y_{j-1}, \ldots, y_{1}, y_{l}, \ldots, y_{j+1}, x_{i+1}, x_{i+2}, \ldots, x_{k} .
$$


We refer to this construction as inserting cycle $Y$ into cycle $X$. Note that we can insert several cycles into $X$ provided that the edges of $X$ that we delete to do the insertion of each are distinct.

Proof of Theorem 1. We assume that $(n, r) \neq(10,4)$ since our construction does not work in this case. However, it is known that $K(10,4)$ is Hamiltonian so this is not a problem. We will show, by induction on $i$, that a cycle containing all vertices of $K_{\{0,1, \ldots, i\}}(n, r)$ can be constructed, containing for each $d \in\{ \pm 1\}^{r-2 i-2}$ an edge $X Y$ with

$$
\begin{aligned}
& X=(d, 0, \underbrace{0, \ldots, 0}_{i}, \pm 1, \underbrace{2, \ldots, 2}_{i}, \pm 1, \underbrace{0, \ldots, 0}_{m-r-1}) \\
& Y=(\bar{d}, \pm 1, \underbrace{2, \ldots, 2}_{i}, 0, \underbrace{0, \ldots, 0}_{i}, \pm 1, \underbrace{0, \ldots, 0}_{m-r-1}) .
\end{aligned}
$$
1 .

When $i=\left\lfloor\frac{r}{2}\right\rfloor$ this gives a Hamilton cycle in $K(n, r)$ and so this claim implies Theorem

Lemma 1 shows that this is true for $i=0$.

Now take $0 \leq i \leq\left\lfloor\frac{r}{2}\right\rfloor$ and suppose that the claim is true for $i$. Let $\mathcal{H}_{i}$ be a cycle in $K_{\{0,1, \ldots, i\}}(n, r)$ as guaranteed by the induction hypothesis. Take a partition of $K_{i+1}(n, r)$ into cycles $C_{1}, \ldots, C_{t}$ as described by Lemma 2 (with $a=i+1$ ). Note that the conditions of this Lemma are met since $\frac{s}{2}=m-r$ and we know that $K\left(2 a+\frac{s}{2}, a\right)$ is either Hamiltonian or $K(5,2)$. By the first part of Lemma 2 each cycle in this partition contains an edge $X Y$ with:

$$
\begin{aligned}
& X=(c, \underbrace{2, \ldots, 2}_{i+1}, \underbrace{0, \ldots, 0}_{i+1}, \underbrace{0, \ldots, 0}_{m-r}) \\
& Y=(\bar{c}, \underbrace{0, \ldots, 0}_{i+1}, \underbrace{2, \ldots, 2}_{i+1}, \underbrace{0, \ldots, 0}_{m-r})
\end{aligned}
$$

for some $c \in\{ \pm 1\}^{r-2 i-2}$, with distinct vectors $c(1), \ldots, c(t)$ playing the role of $c$ in cycles $C_{1}, \ldots, C_{t}$ respectively.

Further, by the induction hypothesis, for each $d \in\{ \pm 1\}^{r-2 i-2}$ there is an edge $X Y$ in $\mathcal{H}_{i}$ with

$$
\begin{aligned}
& X=(d, 0, \underbrace{0, \ldots, 0}_{i}, \pm 1, \underbrace{2, \ldots, 2}_{i}, \pm 1, \underbrace{0, \ldots, 0}_{m-r-1}) \\
& Y=(\bar{d}, \pm 1, \underbrace{2, \ldots, 2}_{i}, 0, \underbrace{0, \ldots, 0}_{i}, \pm 1, \underbrace{0, \ldots, 0}_{m-r-1}) .
\end{aligned}
$$

In particular for each $c(j)$ we can find such an edge with $d=\overline{c(j)}$. These edges can be used to insert each of the cycles $C_{1}, \ldots, C_{t}$ into $\mathcal{H}_{i}$. The fact that the $c(j)$ are distinct 
means that we do not ever have to use the same edge to insert more than one cycle. The result is a cycle containing all vertices of $K_{\{0,1, \ldots, i+1\}}(n, r)$ which we will denote by $\mathcal{H}_{i+1}^{\prime}$.

Now we take a permutation of the pairs $p_{1}, \ldots, p_{m}$ with the properties of Lemma 2 part 2 and apply it to each vertex in $\mathcal{H}_{i+1}^{\prime}$. Note that under this permutation $\mathcal{H}_{i+1}^{\prime}$ is mapped to another cycle containing all vertices of $K_{\{0,1, \ldots, i+1\}}(n, r)$. We denote this new cycle by $\mathcal{H}_{i+1}$. By Lemma 2, for each $d \in\{ \pm 1\}^{r-2 i-2}$ there is an edge $X Y$ in one of the cycles $C_{1}, \ldots, C_{t}$ of the form:

$$
\begin{aligned}
& X=(d, 0, \underbrace{0, \ldots, 0}_{i+1}, \pm 1, \underbrace{2, \ldots, 2}_{i+1}, \pm 1, \underbrace{0, \ldots, 0}_{m-r-1}) \\
& Y=(\bar{d}, \pm 1, \underbrace{2, \ldots, 2}_{i+1}, 0, \underbrace{0, \ldots, 0}_{i+1}, \pm 1, \underbrace{0, \ldots, 0}_{m-r-1}) .
\end{aligned}
$$

These edges are not destroyed by the insertion of $C_{1}, \ldots, C_{t}$ into $\mathcal{H}_{i}$ because the only edges destroyed in this process are of the form $X Y$ with $B(X)=B(Y)$. Hence $\mathcal{H}_{i+1}$ satisfies the properties required and the proof is complete.

\section{Proofs}

Let $\mathcal{B}=B_{1}, \ldots, B_{s}$ be a sequence of $b$-subsets of $[m]$. For each $1 \leq i \leq s$ let $\mathcal{A}^{(i)}=$ $A_{1}^{(i)}, \ldots, A_{t}^{(i)}$ be a sequence of $a$-subsets of $[m] \backslash B_{i}$. We will use these sequences together with a single function $f: B_{1} \rightarrow\{ \pm 1\}$ to construct a sequence $\mathcal{P}=P_{1}, \ldots, P_{s t}$ of $r$ subsets of $[n]$ where $r=2 a+b$ (in fact in all our applications it will be a path in $K(n, r)$ ). We will define the $P_{k}$ by specifying $B\left(P_{k}\right), A\left(P_{k}\right)$ and $f_{P_{k}}$. Firstly, for $1 \leq k \leq$ st let $k=(x-1) t+y$ with $1 \leq x \leq s$ and $1 \leq y \leq t$. We set

$$
B\left(P_{k}\right)=B_{x} ; \quad A\left(P_{k}\right)=A_{y}^{(x)} .
$$

We set $f_{P_{1}}=f$ and given $f_{P_{k-1}}$ define $f_{P_{k}}$ inductively as follows.

If $B_{P_{k}} \backslash B_{P_{k-1}}=\left\{r_{1}, \ldots, r_{l}\right\}, B_{P_{k-1}} \backslash B_{P_{k}}=\left\{s_{1}, \ldots, s_{l}\right\}$ with $r_{1}<r_{2}<\cdots<r_{l}$ and $s_{1}<s_{2}<\cdots<s_{l}$ then

$$
f_{P_{k}}(x)=\left\{\begin{aligned}
-f_{P_{k-1}}(x) & \text { if } k \in B_{P_{k}} \cap B_{P_{k-1}} \\
f_{P_{k-1}}\left(s_{i}\right) & \text { if } x=r_{i} .
\end{aligned}\right.
$$

Note that if $B_{P_{k}}=B_{P_{k-1}}$ then $f_{P_{k}}(x)=-f_{P_{k-1}}(x)$.

We denote the sequence $\mathcal{P}$ by $\mathcal{P}\left(\mathcal{B} ; f ; \mathcal{A}^{(1)}, \ldots, \mathcal{A}^{(s)}\right)$ and refer to this construction as the $\mathcal{P}$ construction.

An important point of the definition of the $f$ is that it is reversible; if $B\left(P_{k-1}\right), B\left(P_{k}\right)$ and $f_{P_{k}}$ are given then $f_{P_{k-1}}$ is uniquely determined by the same rule as above.

We will use this construction to build paths in $K(n, r)$ and so we will need to know when consecutive sets in the sequence are disjoint. The following simple lemma will be used. 
Lemma 3. If $\mathcal{B}, f$ and $\mathcal{A}^{(i)}$ are as above and in addition we have:

1. $A_{1}^{(i)} \cap B_{i-1}=\emptyset$ for all $2 \leq i \leq s$,

2. $A_{t}^{(i)} \cap B_{i+1}=\emptyset$ for all $1 \leq i \leq s-1$,

3. each sequence $\mathcal{A}^{(i)}$ is a path in $K\left([m] \backslash B_{i}, a\right)$ (that is $A_{k}^{(i)} \cap A_{k+1}^{(i)}=\emptyset$ for all $1 \leq i \leq s$, $1 \leq k \leq t-1)$

then $\mathcal{P}\left(\mathcal{B} ; f ; \mathcal{A}^{(1)}, \ldots, \mathcal{A}^{(s)}\right)$ is a path in $K(n, r)$.

Proof. Let $X, Y$ be sets. We have that $X \cap Y=\emptyset$ unless one of the following holds for some $i$ :

1. $(X(i), Y(i))=( \pm 1,2)$ or $(2, \pm 1)$,

2. $(X(i), Y(i))=(2,2)$,

3. $(X(i), Y(i))=(1,1)$ or $(-1,-1)$.

For consecutive elements of $\mathcal{P}\left(\mathcal{B} ; f ; \mathcal{A}^{(1)}, \ldots, \mathcal{A}^{(s)}\right)$ the first of these possibilities is ruled out by conditions 1 and 2 of the Lemma. The second possibility is ruled out by condition 3 of the Lemma. The third possibility is ruled out by the definition of $f$.

For $S$ a finite set we write $G(S, r)$ for the graph with vertex set $S^{(r)}$ and two vertices $A$ and $B$ adjacent if $|A \cap B|=r-1$. Up to isomorphism $G(S, r)$ depends only on the cardinality of $S$ and so for $n \in \mathbb{N}$ we shall write $G(n, r)$ for $G([n], r)$.

Lemma 4. For all $1 \leq r \leq n$ the graph $G(n, r)$ contains:

1. a Hamilton path which starts at the vertex $[r]$, ends at the vertex $[n-r+1, n]$, and contains

$$
[r-1] \cup\{r\},[r-1] \cup\{r+1\},[r-1] \cup\{r+2\}, \ldots,[r-1] \cup\{n\}
$$

as consecutive vertices,

2. a Hamilton cycle which contains

$$
[r-1] \cup\{r\},[r-1] \cup\{r+1\},[r-1] \cup\{r+2\}, \ldots,[r-1] \cup\{n\}
$$

as consecutive vertices.

Where if $n=r$ we interpret the single vertex $[n]$ as being both a Hamilton path and a Hamilton cycle with the required properties. 
Proof. We use induction on $n$. The result clearly holds for $n=2$ (interpreting the single edge $G(2,1)$ as a cycle), We may assume, by part 1 of the inductive hypothesis, that for all $1 \leq k \leq n-r+1$ there is a Hamilton path in $G([k+1, n], r-1)$ from $[k+1, k+r-1]$ to $[n-r+2, n]$. Adding $k$ to each vertex gives a path $P_{k}$ in $G(n, r)$ from $[k, k+r-1]$ to $\{k\} \cup[n-r+2, n]$ containing all the vertices $v \in G(n, r)$ with $\min (v)=k$. Denote the reverse of a path $P$ by $r(P)$. Now the path required by part 1 is given by $P_{1}, r\left(P_{2}\right), P_{3}, r\left(P_{4}\right), \ldots, P_{n-r+1}$, and the cycle required by part 2 is given by $r\left(P_{1}\right), P_{2}, r\left(P_{3}\right), P_{4}, \ldots, P_{n-r+1}$ (note that $P_{n-r+1}$ is a single vertex).

Proof of Lemma 2. Let $b=r-2 a, s=\left(\begin{array}{c}m \\ b\end{array}\right)$ and $t=\left(\begin{array}{c}m-b \\ a\end{array}\right)$.

We use the $\mathcal{P}$ construction to define $2^{b}$ paths in $K(n, r)$ each containing st vertices. These will form the building blocks for our cycles.

We start by taking $\mathcal{B}=B_{1}, \ldots, B_{s}$ to be a Hamilton cycle in $G(m, b)$. By Lemma 4 (and relabelling elements of the ground set) we may take this cycle to have $B_{1}=$ $[1, b-2] \cup\{m-1, m\}$ and $B_{s-k}=[1, b-1] \cup\{m-k\}$ for $m-b \geq k \geq 0$.

We now define sets $F_{i}, L_{i}$ for $1 \leq i \leq s$ inductively as follows:

- $F_{1}=[m-a-1, m-2]$.

- Given $F_{i}$ we take $L_{i}$ to be the lexicographically least element of $[m]^{(a)}$ with

$$
L_{i} \cap\left(F_{i} \cup B_{i} \cup B_{i+1}\right)=\emptyset
$$

(such a set exists since $m-\left|F_{i} \cup B_{i} \cup B_{i+1}\right| \geq m-(a+b+1) \geq r+1-(r-a+1)=a$ ).

- Given $L_{i}$ we take $F_{i+1}$ to be the lexicographically least element of $[m]^{(a)}$ with

$$
F_{i+1} \cap\left(L_{i} \cup B_{i} \cup B_{i+1}\right)=\emptyset
$$

(again such a set exists since $m-\left|L_{i} \cup B_{i} \cup B_{i+1}\right| \geq a$ ).

We claim that $L_{s}=[b, b+a-1]$.

To see this note that for all $t \in[b, b+a-1]$ we have that $t \in B_{s-m+t}$ but $t \notin B_{x}$ for $x>s-m+t$. Hence by construction $t \notin F_{s-m+t+1}$ and, since $L_{x}$ is chosen to be the lexicographically smallest suitable set, $t \in L_{s-m+t+1}$. It follows similarly that for all $s-m+t \leq x \leq s$ we have $t \notin F_{x}$ and $t \in L_{x}$. In particular $L_{s}=[b, b+a-1]$.

The important consequence of this claim is that $F_{1} \cap\left(L_{s} \cup B_{s} \cup B_{1}\right)=\emptyset$ (since $b+$ $a-1<m-a-1)$ and so the property we demand in the construction of the $F_{i}, L_{i}$ holds going "around the corner" from $B_{s}$ to $B_{1}$.

Assuming now that $(m-b, a) \neq(5,2)$ we let $\mathcal{A}^{(i)}=A_{1}^{(i)}, \ldots, A_{t}^{(i)}$ be a Hamilton cycle in $K\left([m] \backslash B_{i}, a\right)$ with $A_{1}^{(i)}=F_{i}$ and $A_{t}^{(i)}=L_{i}$. Such a cycle exists by the hypotheses of the Lemma. Indeed, $K\left([m] \backslash B_{i}, a\right)$ is $K\left(\frac{n}{2}-b, \frac{r-b}{2}\right)$ for some $0 \leq b \leq r-2$ or equivalently is $K\left(r-b+\frac{n-2 r}{2}, \frac{r-b}{2}\right)$ for some $\left\lfloor\frac{r}{2}\right\rfloor \geq \frac{r-b}{2} \geq 1$.

By Lemma 3 , we have that $\mathcal{P}\left(\mathcal{B} ; f ; \mathcal{A}^{(1)}, \ldots, \mathcal{A}^{(s)}\right)=P_{1}, P_{2}, \ldots, P_{s t}$ is a path in $K_{a}(n, r)$ for each of the $2^{b}$ choices for $f: B_{1} \rightarrow\{ \pm 1\}$. Moreover, each choice of $f$ results in a distinct path and (by the reversibility of the definition of $f$ in the $\mathcal{P}$ construction) these $2^{b}$ 
paths partition $K_{a}(n, r)$. Finally, we can construct a bijection from the set of last vertices of these paths to the set of first vertices of these paths by mapping $\left(A\left(P_{s t}\right), B\left(P_{s t}\right), f\right)$ to $\left(A\left(P_{1}\right), B\left(P_{1}\right), g\right)$ where

$$
g(x)=\left\{\begin{aligned}
-f(x) & \text { if } x \in X_{s t} \cap X_{1} \\
f(b-1) & \text { if } x=m-1
\end{aligned}\right.
$$

(Note that $P_{1}=P_{s t} \backslash\{b-1\} \cup\{m-1\}$ so this is the same process as used to construct the $f_{X}$ in the $\mathcal{P}$ construction.)

This results in the paths being joined to form at most $2^{r-2 a}$ cycles $C_{1}, \ldots, C_{k}$ which cover $K(n, r)$.

Finally, we observe that there is an ordering of the pairs $p_{1}, \ldots, p_{m}$ for which we have edges of the form required by part 1 of the Lemma. This follows from the fact that if we permute the pairs $p_{1}, \ldots, p_{m}$ so that there is an edge $X Y$ in $C_{1}$ with $A(X)=$ $[r-2 a+1, r-a], A(Y)=[r-a+1, r], B(X)=B(Y)=[r-2 a]$ then there will be an edge of this form in each of the $C_{i}$. Similarly there is a permutation of $[m]$ resulting in edges of the form required by part 2 of the Lemma. Every possible $d$ occurs because the pattern of $A(X), B(X)$ going round the cycle does not depend on $f_{X}$. That is, if $X$ is followed by $Y$ in some $C_{i}$ then $A(Y), B(Y)$ depend only on $A(X), B(X)$ and not on $f_{X}$.

If $(m-b, a)=(5,2)$ then we use the same method but instead of a Hamilton cycle in $K(5,2)$ use a Hamilton path. This means the choice of $F_{i}$ and $L_{i}$ must be changed. Specifically, given $F_{i}$ we choose $L_{i}$ to be an arbitrary element of $[m]^{(2)}$ with $\left|F_{i} \cap L_{i}\right|=1$ and $L_{i} \cap\left(B_{i} \cup B_{i+1}\right)=\emptyset$. The method for choosing $F_{i}$ is as before. Now there is a Hamilton path in $K\left([m] \backslash B_{i}, 2\right)$ from $F_{i}$ to $L_{i}$ and we use this as our $\mathcal{A}^{(i)}$. We can still choose $L_{s}$ so that $F_{1} \cap\left(L_{s} \cup B_{s} \cup B_{1}\right)$ unless $F_{s}=\{m-5, m-4\}$ or $\{m-3, m-2\}$. We can avoid these possibilities for $F_{s}$ unless $L_{s-1}=\{m-5, m-4\}$ or $\{m-3, m-2\}$. However, there is no possible $F_{s-1}$ for which these are the only choices for $L_{s-1}$ and so we always have a suitable $L_{s-1}, F_{s}, L_{s}$.

Let $L_{1}, \ldots, L_{h}, R_{1}, \ldots, R_{h}$ be pairwise disjoint $k$-sets with $w_{i}, x_{i} \in L_{i}$ and $y_{i}, z_{i} \in R_{i}$. Let $H(k, h)=(V, E)$ be the graph with

$$
V=\bigcup_{i=1}^{h} L_{i} \cup \bigcup_{j=1}^{h} R_{j}
$$

and

$$
E=\bigcup_{i=1}^{h}\left\{l r: l \in L_{i}, r \in R_{i}\right\} \cup \bigcup_{i=1}^{h}\left\{y_{i} w_{i+1}, z_{i} x_{i+1}\right\}
$$

where we identify $w_{h+1}, x_{h+1}$ with $w_{1}, x_{1}$ respectively. We refer to the vertices $w_{i}, x_{i}, y_{i}, z_{i}$ as linking vertices.

So, $H(k, h)$ comprises $h$ pairwise disjoint copies of the complete bipartite graph $K_{k, k}$ arranged in a cycle with two independent edges going from the right-hand part of each $K_{k, k}$ to the left-hand part of its successor around the cycle. 
We also (with a slight abuse of notation) denote by $H^{\prime}(k, h)$ any graph constructed in the same way as $H(k, h)$ but allowing the $L_{i}$ and $R_{i}$ to be $k$-sets or $(k-1)$-sets (but requiring that $\left|L_{i}\right|=\left|R_{i}\right|$ for all $\left.i\right)$.

Lemma 5. For all $h \geq 2$ and $k \geq 3$ each of the graphs $H(k, h)$ and $H^{\prime}(k, h)$ contains a Hamilton cycle. Moreover, if $M$ is a set of independent edges, each lying in one of the complete bipartite graphs induced by $L_{i} \cup R_{i}$ for $1 \leq i \leq h$ and with $w_{1} y_{1}, x_{1} y_{1} \notin M$, then the Hamilton cycles can be chosen so as to include all edges in $M$.

Proof. We start from the linking vertex $y_{1} \in R_{1}$. This is joined to a vertex $w \in L_{2}$. Since $M$ is an independent set of edges and there are 2 linking vertices in $R_{2}$, there is some linking vertex $y \in R_{2}$ with $w y \notin M$. We take a path through all vertices of $L_{2} \cup R_{2}$ which starts at $w$, contains all edges in $M$ which lie in the bipartite graph induced by $L_{2} \cup R_{2}$, and ends at $y$. Continuing inductively we produce a path ending at a linking vertex $z \in R_{h}$. This can be joined to a vertex $x \in L_{1}$ and the cycle can be completed here (using the fact that the edge from $x$ to $y_{1}$ is not in $M$ ).

An identical argument applies to $H^{\prime}(k, h)$.

We will prove Lemma 1 for odd $r$ by finding a copy of $H\left(r+1,2^{r-1}\right)$ in $K_{0}(n, r)$ and specifying a set $M$ of independent edges in it satisfying the conditions of Lemma 5 . By this Lemma we can find a Hamilton cycle $\mathcal{C}$ in this graph containing all the edges in $M$. We then partition the remaining vertices of $K_{0}(n, r)$ into cycles which can be inserted into $\mathcal{C}$ using edges in $M$.

If $r$ is even the strategy is identical except that a copy of $H^{\prime}\left(r+1,2^{r-1}\right)$ must be used.

Proof of Lemma 1. Suppose first that $r$ is odd. Let $V$ be the set of vectors in $\{-1,0,1\}^{m}$ which satisfy:

1. $v_{1}, \ldots, v_{r} \in\{ \pm 1\}$,

2. $v_{r+1}=1$,

3. $v_{r+2}=\cdots=v_{m}=0$,

4. $\left|\left\{i: v_{i}=1\right\}\right| \equiv 0 \bmod 2$.

Note that $|V|=2^{r-1}$.

For $v \in V$ define

$$
\begin{aligned}
& L_{v}=\left\{X \in K_{0}(n, r):\left|\left\{i: 1 \leq i \leq r+1, X(i)=v_{i}\right\}\right|=r\right\}, \\
& R_{v}=\left\{X \in K_{0}(n, r):\left|\left\{i: 1 \leq i \leq r+1, X(i)=-v_{i}\right\}\right|=r\right\} .
\end{aligned}
$$

So $L_{v}$ comprises sets $X$ with a single 0 among the first $r+1$ coordinates and with $X(i)$ agreeing with $v_{i}$ on the remaining $r$, while $R_{v}$ comprises sets $X$ with a single 0 among the first $r+1$ coordinates and with $X(i)$ agreeing with $-v_{i}$ on the remaining $r$. 
The subgraph of $K_{0}(n, r)$ induced by $L_{v} \cup R_{v}$ is isomorphic to $K_{r+1, r+1}$. For example, if $m=4, r=3, v=(+1,-1,-1,+1)$ we have:

$$
\begin{aligned}
& L_{v}=\{(+1,-1,-1,0),(+1,-1,0,+1),(+1,0,-1,+1),(0,-1,-1,+1)\} \\
& R_{v}=\{(-1,+1,+1,0),(-1,+1,0,-1),(-1,0,+1,-1),(0,+1,+1,-1)\} .
\end{aligned}
$$

If $m=7, r=3, v=(+1,-1,-1,+1,0,0,0)$ then $(0,0,0)$ is appended to each of the elements of $L_{v}, R_{v}$ above (and the graph induced is still $K_{4,4}$ ).

The fact that $r$ is odd means that the $L_{v}$ and $R_{v}$ are pairwise disjoint. We will refer to the $K_{r+1, r+1}$ subgraphs described here as $(L, R)$-blocks.

We can order the elements of $V$ cyclically as $v_{1}, v_{2}, \ldots, v_{2^{r-1}}$ so that consecutive elements differ in exactly two coordinates. To achieve this take a cyclic ordering of $\{ \pm 1\}^{r}$ with consecutive elements differing in exactly one coordinate (that is a Hamilton cycle in the hypercube or Gray code). Taking alternate elements of this cycle and appending +1 followed by $m-r-10$ s to each gives the required cyclic ordering. We note now (because we will need it later) that we may choose this ordering so that the coordinate 1 does not change value between $v_{1}$ and $v_{2}$.

If $u$ is the predecessor of $v$ in this ordering then, because $u$ and $v$ differ in only two coordinates, we can find $w, x \in R_{u}$ and $y, z \in L_{v}$ with $w y, x z \in E\left(K_{0}(n, r)\right)$. It follows that we have a copy of $H\left(r+1,2^{r-1}\right)$ in $K_{0}(n, r)$.

For each $c \in\{ \pm 1\}^{r-2}$ choose an edge $X Y$ within some $(L, R)$-block of the form

$$
\begin{aligned}
X & =(c, 0, \pm 1,+1, \underbrace{0, \ldots, 0}_{m-r-1}) \\
Y & =(\bar{c}, \pm 1,0,-1, \underbrace{0, \ldots, 0}_{m-r-1}) .
\end{aligned}
$$

For each such $c$ there are two choices for such an edge, and so we can make our choice avoiding edges in the block induced by $L_{v_{1}} \cup R_{v_{1}}$. Call this set of (necessarily independent) edges $M_{1}$.

If $m=r+1$ then our copy of $H\left(r+1,2^{r-1}\right)$ is a spanning subgraph of $K_{0}(n, r)$. By Lemma 5 there is a Hamilton cycle in this graph containing all edges in $M_{1}$. This satisfies the conditions required by Lemma 1 .

If $m>r+1$ then we need to deal with the vertices in $T=[m]^{(r)} \backslash[r+1]^{(r)}$.

Let $\mathcal{B}=B_{1}, \ldots, B_{l}$ be any ordering of the sets in $T$ and let $\mathcal{A}^{(i)}=\emptyset$ for $1 \leq i \leq l$. Now for each $f: B_{1} \rightarrow\{ \pm 1\}$ we have that $\mathcal{P}\left(\mathcal{B} ; f ; \mathcal{A}^{(1)}, \ldots, \mathcal{A}^{(l)}\right)$ is a path in $K_{0}(n, r)$ containing $l$ vertices. Between them these $2^{b}$ paths contain every vertex in $T$ exactly once. We can join these paths into cycles as in the proof of Lemma 2. That is, we join the last vertex $\left(B_{l}, \emptyset, f\right)$ to the first vertex $\left(B_{1}, \emptyset, g\right)$ where $g$ is constructed from $f, B_{l}, B_{1}$ exactly as $f_{P_{k}}$ is constructed from $f_{P_{k-1}}, B_{k-1}, B_{k}$ in the definition of the $\mathcal{P}$ construction.

We may assume (by choosing $\mathcal{B}$ suitably) that $B_{1}=[3, r+2], B_{2}=\{2\} \cup[4, r+2]$. This means that each cycle contains an edge $\left(B_{1}, \emptyset, f\right)\left(B_{2}, \emptyset, g\right)$. If $f(r+1)=-1$ then pick any edge in the block $L_{v} \cup R_{v}$ with

$$
v=( \pm 1, g(2),-f(3), \ldots,-f(r+1), 0, \ldots, 0) .
$$


If $f(r+1)=1$ then pick any edge in the block $L_{v} \cup R_{v}$ with

$$
v=( \pm 1,-g(2), f(3), \ldots, f(r+1), 0, \ldots, 0) .
$$

Let this set of edges be $M_{2}$. We can choose these edges so that $M_{2} \cap M_{1}=\emptyset, M_{1} \cup M_{2}$ is independent, and the edges in $M_{2}$ are not incident with any linking vertices in the block induced by $L_{v_{1}} \cup R_{v_{1}}$. By Lemma 5 there is a Hamilton cycle in our copy of $H\left(r+1,2^{r-1}\right)$ containing all edges in $M_{1} \cup M_{2}$. Inserting the cycles partitioning $T$ into this using the edges in $M_{2}$ gives a Hamilton cycle in $K_{0}(n, r)$ which satisfies the conditions required by Lemma 1.

If $r$ is even then the same argument holds except for the fact that the $L_{v}$ and $R_{v}$ are no longer pairwise disjoint; for example $R_{(+1,-1,-1,-1,+1)}$ and $L_{(-1,+1,+1,+1,+1)}$ both contain $(+1,-1,-1,-1,0)$. We can get round this by deleting the set $X$ with $X(r+1)=0$ from $L_{v}$ and $R_{v}$ for all $v$ ending with $+1,+1$. This gives $H^{\prime}\left(r+1,2^{r-1}\right)$ as a subgraph of $K_{0}(n, r)$. Notice that we never delete a set which will play the part of $w, x, y, z$ in linking the bipartite blocks since the two coordinates which vary between the $u$ and $v$ associated with consecutive bipartite blocks never include $r+1$. We claim that those vertices in the set

$$
U=\{X: B(X)=[r],|\{i: X(i)=1\}| \equiv 0 \bmod 2\}
$$

have not been included. Indeed, if $X \in U$ then modifying $X$ by setting $X(r+1)$ to be 1 does not produce a vector in $V$. It follows that $X$ is not in any $L_{V}$. Similarly, modifying $X$ by setting $X(r+1)$ to be -1 and negating all coordinates does not produce a vector in $V$ and so $X$ is not in any $R_{v}$.

Notice that $U$ consists of $2^{r-1}$ vertices comprising $2^{r-2}$ pairs $(X, Y)$ with $X(i)=-Y(i)$.

We let $M_{3}$ be the set of all edges $X Y$ within $(L, R)$-blocks with $B(X)=B(Y)=$ $[2, r+2]$. By our choice of ordering of $V$ the edges in $M_{3}$ are not incident with any linking vertices in the block induced by $L_{v_{1}} \cup R_{v_{1}}$. We now choose $M_{1}$ and $M_{2}$ as before, ensuring also that $M_{1}, M_{2}, M_{3}$ are pairwise disjoint and that $M_{1} \cup M_{2} \cup M_{3}$ is an independent set. By Lemma 5 there is a Hamilton cycle in our copy of $H^{\prime}\left(r+1,2^{r-1}\right)$ containing all edges in $M_{1} \cup M_{2} \cup M_{3}$. We insert the pairs of vertices in $U$ into this using the edges in $M_{3}$. Finally, as before, we insert the cycles partitioning $T$ into this using the edges in $M_{2}$. This gives a Hamilton cycle in $K_{0}(n, r)$ satisfying the conditions required by Lemma 1.

\section{References}

[1] N. Biggs, Some odd graph theory, Ann. New York Acad. Sci., 319 (1979), 71-81.

[2] Y-C. Chen, Triangle-free Hamiltonian Kneser graphs, J. Combin. Theory Ser. B 89 (2003), no. 1, 1-16.

[3] I. Shields and C.D. Savage, A note on Hamilton cycles in Kneser graphs, Bull. Inst. Combin. Appl. 40 (2004), 13-22. 